PROCEEDINGS OF THE

AMERICAN MATHEMATICAL SOCIETY

Volume 139, Number 2, February 2011, Pages 597-608

S 0002-9939(2010)10502-6

Article electronically published on July 26, 2010

\title{
POINTWISE ESTIMATES FOR RETRACTIONS ON THE SOLUTION SET TO LIPSCHITZ DIFFERENTIAL INCLUSIONS
}

\author{
ANDRZEJ FRYSZKOWSKI AND TADEUSZ RZEŻUCHOWSKI
}

(Communicated by Yingfei Yi)

Abstract. Denote by $S_{F}^{\prime}(\zeta)$ the set of derivatives of all absolutely continuous solutions of a Lipschitz differential inclusion

$$
\left\{\begin{array}{c}
x^{\prime} \in F(t, x), \quad t \in[0,1]=I, \\
x(0)=\zeta .
\end{array}\right.
$$

It is known that the set $S_{F}^{\prime}(\zeta)$ is an absolute retract. We show the following:

Theorem. For every $\varepsilon>0$ there exists a continuous mapping $r: X \times L^{1} \rightarrow L^{1}$ such that for every $\zeta \in X$ the map $r(\zeta, \cdot)$ is a retraction of $L^{1}$ onto $S_{F}^{\prime}(\zeta)$ and for all $(\zeta, u) \in X \times L^{1}$ and almost all $t \in I$ we have a Filippov type pointwise estimate

$$
\begin{gathered}
|r(\zeta, u)(t)-u(t)| \\
\leq \varepsilon(1+l(t))\|p(\zeta, u)\|+l(t) \int_{0}^{t} e^{m(t)-m(s)} p(\zeta, u)(s) d s+p(\zeta, u)(t),
\end{gathered}
$$

where

$$
p(\zeta, u)(t)=\operatorname{dist}\left(u(t), F\left(t, \zeta+\int_{0}^{t} u(\tau) d \tau\right)\right) \text { a.e. in } I
$$

and the functions $l$ and $m$ are related with the Lipschitz condition.

\section{INTRODUCTION}

We shall consider a differential inclusion in a Banach space $X$ :

$$
\left\{\begin{array}{c}
x^{\prime} \in F(t, x), \quad t \in[0,1]=I, \\
x(0)=\zeta
\end{array}\right.
$$

We assume throughout the paper that the multifunction $F$ satisfies the Lipschitz condition

$$
d_{H}\left(F\left(t, x_{1}\right), F\left(t, x_{2}\right)\right) \leq l(t)\left|x_{1}-x_{2}\right|,
$$

with $l(\cdot)$ integrable, where $d_{H}(\cdot, \cdot)$ stands for the Hausdorff distance.

Received by the editors May 8, 2009 and, in revised form, March 14, 2010.

2010 Mathematics Subject Classification. Primary 34A60, 54C60; Secondary 34A12, 49J53.

Key words and phrases. Differential inclusion, solution set, Filippov Lemma, retraction of the solution set.

(C)2010 American Mathematical Society Reverts to public domain 28 years from publication 
The famous Filippov Lemma [11] (see also [13]) states, in a finite Euclidean space $X$, that for any absolutely continuous function $y$ satisfying

$$
\operatorname{dist}\left(y^{\prime}(t), F(t, y(t))\right) \leq p(t) \text { a.e. in } I \text {, }
$$

where $p(\cdot)$ is integrable, there exists a solution $x$ of (1.1) with the following two properties:

a) $\quad\left|y^{\prime}(t)-x^{\prime}(t)\right| \leq l(t) \int_{0}^{t} e^{m(t)-m(\tau)} p(\tau) d \tau+p(t)$ a.e. in $I$,

$$
\text { where } m(t)=\int_{0}^{t} l(\tau) d \tau
$$

b) $\quad|y(t)-x(t)-(y(0)-\zeta)| \leq \int_{0}^{t} e^{m(t)-m(\tau)} p(\tau) d \tau$.

A similar result holds in arbitrary Banach spaces. Namely, we have the following version of the Filippov Lemma:

For any absolutely continuous function $y$ satisfying

$$
\operatorname{dist}\left(y^{\prime}(t), F(t, y(t))\right) \leq p(t) \text { a.e. in } I,
$$

where $p(\cdot)$ is integrable, and for every $\varepsilon>0$, there exists a solution $x$ of (1.1) with the following two properties:

$$
\begin{aligned}
& \left.a^{\prime}\right)\left|y^{\prime}(t)-x^{\prime}(t)\right| \leq \varepsilon(1+l(t))+l(t) \int_{0}^{t} e^{m(t)-m(\tau)} p(\tau) d \tau+p(t) \text { a.e. in } I, \\
& \left.b^{\prime}\right)|y(t)-x(t)-(y(0)-\zeta)| \leq \varepsilon+\int_{0}^{t} e^{m(t)-m(\tau)} p(\tau) d \tau .
\end{aligned}
$$

On the other hand, the result of Bressan-Cellina-Fryszkowski (BCF) [7] (see also 13]) assures the existence, for every $\varepsilon>0$, of a retraction $r$ from the space of integrable functions $L^{1}=L^{1}(T, X)$ onto the set

$$
S_{F}^{\prime}(\zeta)=\left\{x^{\prime} \in L^{1}: x \text { is a solution of (1.1) }\right\}
$$

such that for each $u \in L^{1}$ we have the estimate

$$
\|r(u)-u\| \leq(1+\varepsilon) \int_{0}^{1} p(\zeta, u)(t) d t
$$

where $p: X \times L_{*}^{1} \rightarrow L^{1}(I, R)$ is a continuous mapping given by

$$
p(\zeta, u)(t)=\operatorname{dist}\left(u(t), F\left(t, \zeta+\int_{0}^{t} u(\tau) d \tau\right)\right) \text { a.e. in } I \text {. }
$$

Taking $y=\zeta+\int_{0}^{t} u(s) d s$ and $x^{\prime}=r(u)$ we recognize the last inequality as a norm estimate for the derivatives in the Filippov Lemma (property $a /$ )), while the pointwise effect in $a /$ ) is missing. Since the methods of showing both the Filippov's and the BCF results are quite close, the lack of the pointwise estimates is somehow striking. The purpose of this paper is to fill this gap. Namely, we show that the construction provided in [7] can be improved in a way to give also a pointwise counterpart of the Filippov Lemma. The method we use combines both the Filippov and BCF techniques and is based on the selection result by A. Fryszkowski and Bressan-Colombo (F\&BC) (see [13], Theorem 42). The paper is organized as follows. In Section 2 we give some introductory remarks and formulate 
the main result. In Section 3 we show some auxiliary lemmas, while the proof of the main result is given in Section 4 .

\section{The MAIN RESUlt}

Let $(X,|\cdot|)$ be a Banach space. The family of all nonempty closed and bounded subsets of $X$ we shall denote by $B C l(X)$. The space $B C l(X)$ is endowed with the Hausdorff distance $d_{H}(A, B)$ given for $A, B \in B C l(X)$ by

$$
d_{H}(A, B)=\inf \left\{r: A \subset B^{r}, B \subset A^{r}\right\},
$$

where $A^{r}=\{x: \operatorname{dist}(x, A)<r\}$ and $\operatorname{dist}(x, A)=\inf \{|x-a|: a \in A\}$.

Consider in $X$ a differential inclusion

$$
\left\{\begin{array}{c}
x^{\prime} \in F(t, x), \quad t \in[0,1]=I, \\
x(0)=\zeta .
\end{array}\right.
$$

We impose on the multifunction $F: I \times X \rightsquigarrow B C l(X)$ the following conditions:

i) $F$ is $\mathcal{L} \otimes \mathcal{B}(X)$ - measurable in $(t, x)$;

ii) there exists an integrable function $l$ such that for every $x_{1}, x_{2} \in X$ the inequality

$$
d_{H}\left(F\left(t, x_{1}\right), F\left(t, x_{2}\right)\right) \leq l(t)\left|x_{1}-x_{2}\right|
$$

holds a.e. in $I$.

Let $\mu_{0}$ be an absolutely continuous measure on $I$ defined by

$$
\frac{d \mu_{0}}{d t}=e^{-2 m(t)}, \quad m(t)=\int_{0}^{t} l(\tau) d \tau .
$$

In the space of integrable functions $u:[0,1] \rightarrow X$ we consider the norm

$$
\|u\|_{*}=\int_{0}^{1}|u(t)| \mu_{0}(d t)=\int_{0}^{1}|u(t)| e^{-2 m(t)} d t .
$$

We shall denote this space by $L_{*}^{1}=L_{*}^{1}(I, X)$, while by $L^{1}=L^{1}(I, X)$ we mean the standard space of integrable functions (equivalent classes). It is quite obvious that the topologies in $L_{*}^{1}$ and in $L^{1}$ are the same.

For $u \in L_{*}^{1}, \zeta \in X$ and $t \in I$ we put

$$
\mathcal{R}(\zeta, u)(t)=\zeta+\int_{0}^{t} u(\tau) d \tau
$$

By a solution of (2.1) we mean an absolutely continuous function $x=\mathcal{R}(\zeta, u) \in$ $A C(I, X)$ such that

$$
u(t) \in F(t, \mathcal{R}(\zeta, u)(t)) \text { a.e. in } I .
$$

We shall denote by $S_{F}(\zeta)$ the set of all solutions of (2.1). In other words, the function $x=\mathcal{R}(\zeta, u)$ is a solution of (2.1) if $u$ is a fixed point of the multivalued mapping

$$
\Phi(\zeta, u)=\left\{v \in L_{*}^{1}(I, X): v(t) \in F(t, \mathcal{R}(\zeta, u)(t)) \text { a.e. in } I\right\},
$$

i.e. $u \in \Phi(\zeta, u)$. 
Most properties of the solution sets are consequences of the properties of the set

$$
\mathcal{F} i x(\Phi(\zeta, \cdot))=\{u: u \in \Phi(\zeta, u)\}
$$

of fixed points of $\Phi(\zeta, \cdot)$. The topological structure of the set $S_{F}(\zeta)$ is well recognized (see [2], [7, [5], 16], 18]). In particular, it is known that each set

$$
S_{F}^{\prime}(\zeta)=\left\{x^{\prime}: x \in S_{F}(\zeta)\right\}
$$

is a retract of $\left.L_{*}^{1}(I, X)(7]\right)$. Moreover, retractions can be chosen that depend on $\zeta$ in a continuous way. The construction of such retractions is connected with the Filippov Lemma and establishes some norm estimates in $L_{*}^{1}(I, X)$. But the Filippov Lemma also gives some pointwise estimates for the solutions, while a similar estimate for the retraction was unknown till now. The purpose of this paper is to present such a construction of a retraction which removes this gap. Namely, we shall demonstrate the following result:

Theorem 1. For every $\varepsilon>0$ there exists a continuous function $r: X \times L_{*}^{1} \rightarrow L_{*}^{1}$ such that for every $\zeta \in X$ the mapping $r(\zeta, \cdot)$ is a retraction of $L_{*}^{1}$ onto $S_{F}^{\prime}(\zeta)$ and for all $(\zeta, u) \in X \times L_{*}^{1}$ we have the estimates

$$
\leq \varepsilon(1+l(t))\|p(\zeta, u)\|_{*}+l(t) \int_{0}^{t} e^{m(t)-m(s)} p(\zeta, u)(s) d s+p(\zeta, u)(t) \quad \text { a.e. in } I,
$$
where $p$ is given by (1.2),

and

ii)

$$
\|r(\zeta, u)-u\|_{*} \leq(1+\varepsilon)\|p(\zeta, u)\|_{*} .
$$

The construction and techniques used are close to Filippov's arguments and are based on the Fryszkowski [12] and Bressan-Colombo (F\&BC) [6] (see also [13]) selection theorem.

\section{Some Lemmas}

The required retraction occurs as a limit of iterations $k^{n}(\zeta, u)$ of a properly chosen continuous selection $k(\zeta, u)$ of the mapping

$$
\Phi(\zeta, u)=\left\{v \in L_{*}^{1}(I, X): v(t) \in F(t, \mathcal{R}(\zeta, u)(t)) \text { a.e. in } I\right\} .
$$

We shall proceed in a few steps. Put $S=\left\{(\zeta, u) \in X \times L_{*}^{1}:\|p(\zeta, u)\|_{*}>0\right\}$, where $p$ is given by (1.2). Then $S$ is open and for any given $\delta>0$ the multivalued mapping

$$
K(\zeta, u)=c l\left\{v \in \Phi(\zeta, u):|v(t)-u(t)|<p(\zeta, u)(t)+\delta\|p(\zeta, u)\|_{*}\right\}
$$

is l.s.c. with closed, decomposable values. So, by the Fryszkowski [12] and BressanColombo [6] Selection Theorem (F\&BC) (see also [13]) the mapping $K(\zeta, u)$ admits a continuous selection $k: X \times S \rightarrow L_{*}^{1}$. Therefore for all $(\zeta, u) \in X \times S$ we have

$$
k(\zeta, u)(t) \in F(t, \mathcal{R}(\zeta, u)(t)) \text { a.e. in } I
$$

and

$$
|k(\zeta, u)(t)-u(t)| \leq p(\zeta, u)(t)+\delta\|p(\zeta, u)\|_{*} \text { a.e. in } I .
$$

This selection can be extended to a continuous mapping defined on $L_{*}^{1}$ by setting

$$
k(\zeta, u)=u,
$$


whenever $p(\zeta, u)=0$. Indeed, it is enough to check that

$$
k\left(\zeta_{n}, u_{n}\right) \longrightarrow u
$$

for arbitrary $\left(\zeta_{n}, u_{n}\right) \longrightarrow(\zeta, u)$ with $p(\zeta, u)=0$ and $\left\|p\left(\zeta_{n}, u_{n}\right)\right\|_{*}>0$. Then, by (3.2), we have

$$
\left\|k\left(\zeta_{n}, u_{n}\right)-u_{n}\right\|_{*} \leq(1+\delta)\left\|p\left(\zeta_{n}, u_{n}\right)\right\|_{*} .
$$

Hence, by continuity of $p(\cdot, \cdot)$, the latter inequality yields

$$
k\left(\zeta_{n}, u_{n}\right) \longrightarrow u=k(\zeta, u),
$$

proving our claim.

Let us summarize: For every $\delta>0$ there exists such a continuous mapping $k: X \times L_{*}^{1} \rightarrow L_{*}^{1}$ that for all $(\zeta, u) \in X \times L_{*}^{1}$ we have

$$
k(\zeta, u)(t) \in F(t, \mathcal{R}(\zeta, u)(t)) \quad \text { a.e. in } I
$$

and

$$
|k(\zeta, u)(t)-u(t)| \leq p(\zeta, u)(t)+\delta\|p(\zeta, u)\|_{*} \text { a.e. in } I .
$$

Fix $(\zeta, u) \in X \times L_{*}^{1}$ and denote

$$
p_{n}(t)=p\left(\zeta, k^{n}(\zeta, u)\right)(t) \quad \text { and } \quad a_{n}=\left\|p\left(\zeta, k^{n}(\zeta, u)\right)\right\|_{*},
$$

where $k^{n}(\zeta, u)=\underbrace{k(\zeta, k(\zeta, k(\zeta, \ldots) \ldots))}_{n \text { times }}$ stands for the $n$-th iteration of $k(\zeta, u)$ with respect to the second variable. Then by hypotheses $(i)-(i v)$ we conclude that a.e. in $I$ the recurrence

$$
0 \leq p_{n+1}(t) \leq l(t) \int_{0}^{t}\left(p_{n}(s)+\delta a_{n}\right) d s
$$

holds. Indeed, we proceed a.e. in $I$ in the following way:

$$
\begin{aligned}
0 & \leq p_{n+1}(t)=p\left(\zeta, k^{n+1}(\zeta, u)\right)(t)=\operatorname{dist}\left(k^{n+1}(\zeta, u), F\left(t, \mathcal{R}\left(k^{n+1}(\zeta, u)\right)(t)\right)\right) \\
& \leq d_{H}\left(F\left(t, \mathcal{R}\left(k^{n}(\zeta, u)\right)(t)\right), F\left(t, \mathcal{R}\left(k^{n+1}(\zeta, u)\right)(t)\right)\right) \\
& \leq l(t)\left|\mathcal{R}\left(k^{n+1}(\zeta, u)\right)(t)-\mathcal{R}\left(k^{n}(\zeta, u)\right)(t)\right| \\
& \leq l(t) \int_{0}^{t}\left|\left(k\left(k^{n}(\zeta, u)\right)\right)(s)-\left(k^{n}(\zeta, u)\right)(s)\right| d s \leq l(t) \int_{0}^{t}\left(p_{n}(s)+\delta a_{n}\right) d s .
\end{aligned}
$$

In further consideration we need the following:

Lemma 1. Let the functions $p_{n} \in L_{*}^{1}, n=0,1,2, \ldots$ satisfy a.e. in $I$ the inequalities

$$
0 \leq p_{n+1}(t) \leq l(t) \int_{0}^{t}\left(p_{n}(s)+\delta a_{n}\right) d s
$$

assuming that $a_{n} \geq 0, n=0,1,2, \ldots$, and $\delta>0$ are given. Then the inequalities

$$
p_{n+1}(t) \leq l(t)\left(\sum_{i=0}^{n} \delta a_{i} \frac{[m(t)]^{n-i}}{(n-i) !}+\int_{0}^{t}\left(\frac{[m(t)-m(s)]^{n}}{n !} p_{0}(s)\right) d s\right)
$$

hold a.e. in $I$. 
Proof. We apply the induction. For $n=0$ we have from the given condition that

$$
p_{1}(t) \leq l(t) \int_{0}^{t}\left(p_{0}(s)+\delta a_{0}\right) d s \leq l(t)\left(\delta a_{0} \frac{[m(t)]^{0}}{0 !}+\int_{0}^{t} \frac{[m(t)-m(s)]^{0}}{0 !} p_{0}(s) d s\right) .
$$

Assume that the required inequality holds for all $i \leq n+1$. Then for $n+2$ we have, integrating by parts,

$$
\begin{gathered}
p_{n+2}(t) \leq l(t) \int_{0}^{t}\left(p_{n+1}(s)+\delta a_{n+1}\right) d s \\
\leq l(t) \int_{0}^{t}\left\{m^{\prime}(s)\left(\sum_{i=0}^{n} \delta a_{i} \frac{[m(s)]^{n-i}}{(n-i) !}+\int_{0}^{s}\left(\frac{[m(s)-m(x)]^{n}}{n !} p_{0}(x)\right) d x\right)+\delta a_{n+1}\right\} d s \\
\leq l(t)\left\{\int_{0}^{t}\left(\sum_{i=0}^{n} \delta a_{i} \frac{[m(s)]^{n+1-i}}{(n+1-i) !}+\int_{0}^{s}\left(\frac{[m(s)-m(x)]^{n+1}}{(n+1) !} p_{0}(x)\right) d x\right)^{\prime} d s+\delta a_{n+1}\right\} \\
\leq l(t)\left(\sum_{i=0}^{n+1} \delta a_{i} \frac{[m(t)]^{n+1-i}}{(n+1-i) !}+\int_{0}^{t}\left(\frac{[m(t)-m(x)]^{n+1}}{(n+1) !} p_{0}(x)\right) d x\right) .
\end{gathered}
$$

This ends the proof.

Applying lemma for $a_{n}$ and $p_{n}$ given by (3.4) and integrating by parts, one can see that

$$
\begin{gathered}
a_{n+1}=\left\|p_{n+1}\right\|_{*}=\int_{0}^{1} e^{-2 m(t)} p_{n+1}(t) d t \\
\leq \int_{0}^{1}\left\{\left(\frac{e^{-2 m(t)}}{-2}\right)^{\prime}\left(\sum_{i=0}^{n} \delta a_{i} \frac{[m(t)]^{n-i}}{(n-i) !}+\int_{0}^{t}\left(\frac{[m(t)-m(s)]^{n}}{n !} p_{0}(s)\right) d s\right)\right\} d t \\
\leq \frac{1}{2} \delta a_{n}+\int_{0}^{1}\left\{\left(\frac{e^{-2 m(t)}}{2}\right)\left(\sum_{i=0}^{n} \delta a_{i} \frac{[m(t)]^{n-i}}{(n-i) !}+\int_{0}^{t}\left(\frac{[m(t)-m(s)]^{n}}{n !} p_{0}(s)\right) d s\right)^{\prime}\right\} d t \\
\leq \frac{1}{2} \delta a_{n}+\int_{0}^{1}\left\{\left(\frac{e^{-2 m(t)}}{-4}\right)^{\prime}\left(\sum_{i=0}^{n-1} \delta a_{i} \frac{[m(t)]^{n-i-1}}{(n-i-1) !} \int_{0}^{t}\left(\frac{[m(t)-m(s)]^{n-1}}{(n-1) !} p_{0}(s)\right) d s\right)\right\} d t \\
\leq \frac{1}{2} \delta a_{n}+\frac{1}{2^{2}} \delta a_{n-1} \\
+\int_{0}^{1}\left\{\left(\frac{e^{-2 m(t)}}{2^{2}}\right)\left(\sum_{i=0}^{n-2} \delta a_{i} \frac{[m(t)]^{n-i-2}}{(n-i-2) !}+\int_{0}^{t}\left(\frac{[m(t)-m(s)]^{n-2}}{(n-2) !} p_{0}(s)\right) d s\right)^{\prime}\right\} d t .
\end{gathered}
$$


Continuing this procedure we end up with the recurrence

$$
0 \leq a_{n+1} \leq \delta\left(\sum_{i=0}^{n} \frac{1}{2^{i+1}} a_{n-i}\right) .
$$

Lemma 2. Fix $\delta>0$. Let $\left\{a_{n}\right\}_{n=0}^{\infty}$ be a sequence satisfying, for each $n=0,1, \ldots$, the recurrence inequality

$$
0 \leq a_{n+1} \leq \delta\left(\sum_{i=0}^{n} \frac{1}{2^{i+1}} a_{n-i}\right) .
$$

Then for all $n=1,2, \ldots$ we have

$$
a_{n} \leq \frac{\delta}{1+\delta}\left(\frac{1+\delta}{2}\right)^{n} a_{0} .
$$

Proof. Denoting $b_{n}=2^{n} a_{n}$ we can read the given condition as

$$
0 \leq b_{n+1} \leq \delta\left(\sum_{i=0}^{n} b_{i}\right)
$$

while our claim is

$$
b_{n} \leq \delta(1+\delta)^{n-1} b_{0} .
$$

We shall use the induction argument.

1. For $n=1$ our claim is just the hypothesis $b_{1} \leq \delta b_{0}$.

2. Assuming that the required estimation holds for all indices $i \leq n$ we proceed for $n+1$ as follows:

$$
0 \leq b_{n+1} \leq \delta\left(b_{0}+\sum_{i=1}^{n} b_{i}\right) \leq \delta\left(1+\sum_{i=1}^{n} \delta(1+\delta)^{i-1}\right) b_{0}=\delta(1+\delta)^{n} b_{0} .
$$

This ends the proof.

Applying both Lemmas 1 and 2 we get the following:

Lemma 3. Within the previous notation we have for each $n=0,1,2, \ldots$, the inequalities

$$
\begin{aligned}
& p_{n+1}(t)=p\left(\zeta, k^{n+1}(\zeta, u)\right)(t) \\
& \leq l(t)\left(\delta\|p(\zeta, u)\|_{*}\left(\frac{[2 m(t)]^{n}}{n !}+\frac{\delta e^{2 m(t)}}{1+\delta}\left(\frac{1+\delta}{2}\right)^{n}\right)\right. \\
&\left.\quad+\int_{0}^{t}\left(\frac{[m(t)-m(s)]^{n}}{n !} p(\zeta, u)(s)\right) d s\right)
\end{aligned}
$$

and for each $n=1,2, \ldots$

$$
a_{n}=\left\|p\left(\zeta, k^{n}(\zeta, u)\right)\right\|_{*} \leq \frac{\delta}{1+\delta}\left(\frac{1+\delta}{2}\right)^{n}\|p(\zeta, u)\|_{*} .
$$

Proof. The second inequality is just Lemma 2. 
For the first inequality we conclude, by Lemmas 1 and 2, that

$$
\begin{aligned}
& p_{n+1}(t) \leq l(t)\left(\sum_{i=0}^{n} \delta a_{i} \frac{[m(t)]^{n-i}}{(n-i) !}+\int_{0}^{t}\left(\frac{[m(t)-m(s)]^{n}}{n !} p_{0}(s)\right) d s\right) \\
& \leq l(t)\left(\delta a_{0} \frac{[m(t)]^{n}}{n !}+\delta\left(\sum_{i=0}^{n-1} \frac{\delta}{1+\delta}\left(\frac{1+\delta}{2}\right)^{n-i} \frac{[m(t)]^{i}}{i !}\right)\right. \\
& \left.+\int_{0}^{t}\left(\frac{[m(t)-m(s)]^{n}}{n !} p_{0}(s)\right) d s\right) \\
& \leq l(t)\left(\delta a_{0}\left(\frac{[m(t)]^{n}}{n !}+\frac{\delta}{1+\delta}\left(\frac{1+\delta}{2}\right)^{n} \sum_{i=0}^{n-1} \frac{\left(\frac{2 m(t)}{1+\delta}\right)^{i}}{i !}\right)\right. \\
& \left.+\int_{0}^{t}\left(\frac{[m(t)-m(s)]^{n}}{n !} p_{0}(s)\right) d s\right) \\
& \leq l(t)\left(\delta\|p(\zeta, u)\|_{*}\left(\frac{[2 m(t)]^{n}}{n !}+\frac{\left.\delta e^{2 m(t)}\left(\frac{1+\delta}{2}\right)^{n}\right)}{1+\delta}\left(\frac{[m(t)-m(s)]^{n}}{n !} p(\zeta, u)(s)\right) d s\right) .\right.
\end{aligned}
$$

This ends the proof.

As a conclusion from the above considerations we get

Proposition 1. Within the previous notation we have, for all $n \in N$, that a.e. in I,

$$
\begin{gathered}
\left|k^{n+1}(\zeta, u)(t)-k^{n}(\zeta, u)(t)\right| \\
\leq l(t) \delta\|p(\zeta, u)\|_{*}\left(\frac{[2 m(t)]^{n-1}}{(n-1) !}+\frac{\delta e^{2 m(t)}}{1+\delta}\left(\frac{1+\delta}{2}\right)^{n-1}\right) \\
+l(t) \int_{0}^{t} \frac{[m(t)-m(s)]^{n-1}}{(n-1) !} p(\zeta, u)(s) d s+\frac{\delta^{2}}{1+\delta}\left(\frac{1+\delta}{2}\right)^{n}\|p(\zeta, u)\|_{*}
\end{gathered}
$$

and

$$
\left\|k^{n+1}(\zeta, u)-k^{n}(\zeta, u)\right\|_{*} \leq \delta\left(\frac{1+\delta}{2}\right)^{n}\|p(\zeta, u)\|_{*} .
$$

Proof. The second inequality can easily be derived from Lemma 3 and (3.3), since

$$
\left\|k^{n+1}(\zeta, u)-k^{n}(\zeta, u)\right\|_{*} \leq(1+\delta)\left\|p\left(\zeta, k^{n}(\zeta, u)\right)\right\|_{*} \leq \delta\left(\frac{1+\delta}{2}\right)^{n}\|p(\zeta, u)\|_{*} .
$$


For the first inequality we have for a.a. $t \in I$,

$$
\begin{gathered}
\left|k^{n+1}(\zeta, u)(t)-k^{n}(\zeta, u)(t)\right|=\left|k\left(\zeta, k^{n}(\zeta, u)\right)(t)-k^{n}(\zeta, u)(t)\right| \\
\leq p\left(\zeta, k^{n}(\zeta, u)\right)(t)+\delta\left\|p\left(\zeta, k^{n}(\zeta, u)\right)\right\|_{*}=p_{n}(t)+\delta a_{n} \\
\leq l(t) \delta\|p(\zeta, u)\|_{*}\left(\frac{[2 m(t)]^{n-1}}{(n-1) !}+\frac{\delta e^{2 m(t)}}{1+\delta}\left(\frac{1+\delta}{2}\right)^{n-1}\right) \\
+l(t) \int_{0}^{t} \frac{[m(t)-m(s)]^{n-1}}{(n-1) !} p(\zeta, u)(s) d s+\frac{\delta^{2}}{1+\delta}\left(\frac{1+\delta}{2}\right)^{n}\|p(\zeta, u)\|_{*} .
\end{gathered}
$$

\section{Proof of the Main Result}

Proof of Theorem 1. Now fix $\varepsilon>0$ and take $\delta \in(0,1)$ such that

$$
\max \left\{\delta e^{2 m(1)}\left(1+\frac{2 \delta}{1-\delta^{2}}\right), \frac{2 \delta}{1-\delta}\right\}<\varepsilon .
$$

Using Proposition 1 we shall show first that $\left\{k^{n}(\zeta, u)\right\}$ are locally bounded Cauchy sequences as well pointwisely, as in $L_{*}^{1}$. Indeed, we can proceed for $j, n \in N$ and a.e. in $I$ as follows:

$$
\begin{aligned}
\mid & k^{n+j+1}(\zeta, u)(t)-k^{n}(\zeta, u)(t)\left|\leq \sum_{i=0}^{j}\right| k^{n+i+1}(\zeta, u)(t)-k^{n+i}(\zeta, u)(t) \mid \\
\leq & \delta l(t)\|p(\zeta, u)\|_{*}\left(\sum_{i=0}^{j} \frac{[2 m(t)]^{n+i-1}}{(n+i-1) !}+\frac{\delta e^{2 m(t)}}{1+\delta} \sum_{i=0}^{j}\left(\frac{1+\delta}{2}\right)^{n+i-1}\right) \\
+l(t) \int_{0}^{t} & \left(\sum_{i=0}^{j} \frac{[m(t)-m(s)]^{n+i-1}}{(n+i-1) !}\right) p(\zeta, u)(s) d s+\frac{\delta^{2}\|p(\zeta, u)\|_{*}}{1+\delta} \sum_{i=0}^{j}\left(\frac{1+\delta}{2}\right)^{n+i} \\
& \leq \delta l(t)\|p(\zeta, u)\|_{*}\left(\sum_{i=n-1}^{n+j-1} \frac{[2 m(t)]^{i}}{i !}+\frac{\delta e^{2 m(t)}}{1+\delta} \sum_{i=n-1}^{n+j-1}\left(\frac{1+\delta}{2}\right)^{i}\right) \\
+l(t) & \int_{0}^{t}\left(\sum_{i=n-1}^{n+j-1} \frac{[m(t)-m(s)]^{i}}{i !}\right) p(\zeta, u)(s) d s+\frac{\delta^{2}\|p(\zeta, u)\|_{*}}{1+\delta} \sum_{i=n}^{n+j}\left(\frac{1+\delta}{2}\right)^{i} \\
& \leq \delta l(t)\|p(\zeta, u)\|_{*}\left(\sum_{i=n-1}^{\infty} \frac{[2 m(t)]^{i}}{i !}+\frac{2 \delta e^{2 m(t)}}{1-\delta^{2}}\left(\frac{1+\delta}{2}\right)^{n-1}\right)^{t} \\
& \int_{0}^{t}\left(\sum_{i=n-1}^{\infty} \frac{[m(t)-m(s)]^{i}}{i !}\right) p(\zeta, u)(s) d s+\frac{2 \delta^{2}\|p(\zeta, u)\|_{*}}{1-\delta^{2}}\left(\frac{1+\delta}{2}\right)^{n} .
\end{aligned}
$$

Summarizing, we have for all $j, n \in N$ and a.e. in $I$ the inequalities

$$
\left|k^{n+j+1}(\zeta, u)(t)-k^{n}(\zeta, u)(t)\right| \leq r_{n}(t)
$$


where

$$
\begin{array}{r}
r_{n}(t)=\delta l(t)\|p(\zeta, u)\|_{*}\left(\sum_{i=n-1}^{\infty} \frac{[2 m(t)]^{i}}{i !}+\frac{2 \delta e^{2 m(t)}}{1-\delta^{2}}\left(\frac{1+\delta}{2}\right)^{n-1}\right) \\
+l(t) \int_{0}^{t}\left(\sum_{i=n-1}^{\infty} \frac{[m(t)-m(s)]^{i}}{i !}\right) p(\zeta, u)(s) d s+\frac{2 \delta^{2}\|p(\zeta, u)\|_{*}}{1-\delta^{2}}\left(\frac{1+\delta}{2}\right)^{n} .
\end{array}
$$

Since the integrable functions $r_{n}$ converge a.a. in $I$ to 0 , the latter shows the pointwise Cauchy property.

The Cauchy property in norm can be concluded from the inequalities

$$
\begin{gathered}
\left\|k^{n+j+1}(\zeta, u)-k^{n}(\zeta, u)\right\|_{*} \leq \sum_{i=0}^{j}\left\|k^{n+i+1}(\zeta, u)(t)-k^{n+i}(\zeta, u)(t)\right\|_{*} \\
\leq \sum_{i=0}^{j} \delta\left(\frac{1+\delta}{2}\right)^{n+i}\|p(\zeta, u)\|_{*} \leq \frac{2 \delta}{1-\delta}\left(\frac{1+\delta}{2}\right)^{n}\|p(\zeta, u)\|_{*} .
\end{gathered}
$$

Observe now that the sequence $\left\{k^{n}(\zeta, u)\right\}$ is locally bounded in $L_{*}^{1}$ and thus it is convergent to a continuous mapping $r(\zeta, u): X \times L^{1} \rightarrow L_{*}^{1}$. For this function we have by (4.2) the following estimates a.e. in I:

$$
\left|r(\zeta, u)(t)-k^{n}(\zeta, u)(t)\right| \leq \alpha_{n}(t),
$$

where functions $\alpha_{n}$ are given by (4.3), and

$$
\left\|r(\zeta, u)-k^{n}(\zeta, u)\right\|_{*} \leq \frac{2 \delta}{1-\delta}\left(\frac{1+\delta}{2}\right)^{n}\|p(\zeta, u)\|_{*} .
$$

In particular for $n=1$,

$$
\begin{gathered}
|r(\zeta, u)(t)-k(\zeta, u)(t)| \leq \alpha_{1}(t) \\
=\left[\delta l(t) e^{2 m(t)}\left(1+\frac{2 \delta}{1-\delta^{2}}\right)+\frac{\delta^{2}}{1-\delta}\right]\|p(\zeta, u)\|_{*}+l(t) \int_{0}^{t} e^{m(t)-m(s)} p(\zeta, u)(s) d s
\end{gathered}
$$

and

$$
\|r(\zeta, u)-k(\zeta, u)\|_{*} \leq \frac{\delta(1+\delta)}{1-\delta}\|p(\zeta, u)\|_{*} .
$$

The latter together with (3.1) and (3.3) yields

$$
\begin{gathered}
|r(\zeta, u)(t)-u(t)| \leq|r(\zeta, u)(t)-k(\zeta, u)(t)|+p(\zeta, u)(t)+\delta\|p(\zeta, u)\|_{*} \\
\leq\left(\frac{\delta}{1-\delta}+l(t) \delta e^{2 m(1)}\left(1+\frac{2 \delta}{1-\delta^{2}}\right)\right)\|p(\zeta, u)\|_{*} \\
\quad+l(t) \int_{0}^{t} e^{m(t)-m(s)} p(\zeta, u)(s) d s+p(\zeta, u)(t)
\end{gathered}
$$

and

$$
\|r(\zeta, u)-u\|_{*} \leq\left(\frac{\delta(1+\delta)}{1-\delta}+(1+\delta)\right)\|p(\zeta, u)\|_{*}=\left(1+\frac{2 \delta}{1-\delta}\right)\|p(\zeta, u)\|_{*} .
$$


Thus, applying (4.1), we have

$$
\begin{aligned}
& |r(\zeta, u)(t)-u(t)| \leq \varepsilon(1+l(t))\|p(\zeta, u)\|_{*} \\
+ & l(t) \int_{0}^{t} e^{m(t)-m(s)} p(\zeta, u)(s) d s+p(\zeta, u)(t)
\end{aligned}
$$

and

$$
\|r(\zeta, u)-u\|_{*} \leq(1+\varepsilon)\|p(\zeta, u)\|_{*}
$$

Observe now that

$$
k^{n+1}(\zeta, u)(t) \in F\left(t, \mathcal{R}\left(k^{n}(\zeta, u)\right)(t)\right) \quad \text { a.e. in } I .
$$

Therefore

$$
r(\zeta, u)(t) \in F(t, \mathcal{R}(r(\zeta, u))(t)) \text { a.e. in } I,
$$

which means that for each $u \in L^{1}$ the function $x(\zeta, u)=\mathcal{R}(r(\zeta, u))$ is a solution of (2.1). Moreover, if $x=\mathcal{R}(\zeta, u)$ is a solution of (2.1), then $p(\zeta, u)=0$ and thus $r(\zeta, u)=u$. So $r(\zeta, u)$ is a required mapping, and this ends the proof.

\section{REFERENCES}

[1] Antosiewicz, H., Cellina, A., Continuous selections and differential relations, J. Diff. Eq. 19 (1975), 386-398. MR0430368 (55:3373)

[2] Anroszajn, N., Le correspondant topologique de l'unicité dans la théorie des équations différentielles, Ann. Math. 43 (1942), 730-738. MR0007195 (4:100e)

[3] Aubin, J. P., Cellina, A., Differential inclusions, Springer-Verlag, Berlin, 1984. MR755330 (85j:49010)

[4] Aubin, J. P., Frankowska, H., Set-Valued Analysis, Birkhäuser, Boston, Basel, Berlin, 1990. MR:1048347 (91d:49001)

[5] Bartuzel, G., Fryszkowski, A., A class of retracts in $L^{p}$ with some applications to differential inclusions, Discussiones Math. 22 (2001), 213-224; MR1989312 (2004e:35243)

[6] Bressan, A., Colombo, G., Extensions and selections of maps with decomposable values, Studia Math. 90 (1988), 69-86. MR947921 (89j:54021)

[7] Bressan, A., Cellina, A., Fryszkowski, A., A class of absolute retracts in spaces of integrable functions, Proceedings of American Math. Soc. 112 (1991), 413-418. MR1045587(91i:47076)

[8] Cellina, A., On the set of solutions to Lipschitzian differential inclusions, Diff. and Int. Equations 1 (1988), 495-500. MR.945823 (89d:34023)

[9] Cellina, A., Ornelas, A., Representation of the attainable set for Lipschitzian differential inclusions, Rocky Mountain J. of Math. 22 (1992), 117-124. MR.1159946 (93b:34028)

[10] Colombo, R. M., Fryszkowski, A., Rzeżuchowski, T., Staicu, V., Continuous selection of solution sets of Lipschitzian differential inclusions, Funkcialaj Ekvacioj 34 (1991), 321-330. MR.1130468 (93i:34022)

[11] Filippov, A. F., Classical solutions of differential equations with multi-valued right-hand side, Vestnik Moskov. Univ. Ser. I Mat. Meh. 22 (1967), 16-26. [English translation: SIAM J. Control. 5, 1967, 609-621.] MR0214834 (35:5683) MR0220995 (36:4047)

[12] Fryszkowski, A., Continuous selections for a class of non-convex multivalued maps, Studia Math. 75 (1983), 163-174. MR730018 (85j:54022)

[13] Fryszkowski, A., Fixed point theory for decomposable sets, Kluwer Academic Publishers, Dordrecht, 2004, Topological Fixed Point Theory, vol. 2, 1-206. MR2084131 (2005k:47116)

[14] Górniewicz, L., Marano, S. A., Ślosarski, M., Fixed point of contractive multivalued maps, Proc. Amer. Math. Soc. 124 (1996), 2675-2683. MR.1317038 (96k:54067)

[15] Hu, Sh., Papageorgiou, N. S., Handbook of Multivalued Analysis, vol. I, Kluwer, 1997. MR1485775 (98k:47001)

[16] Nasselli Riccieri, O., $\mathcal{A}$-fixed points of multi-valued contractions, J. Math. Anal. Appl. 135 (1988), 406-418. MR967219 (90a:54113) 
[17] Repovš, D., Semenov, P. V., Continuous selections of multivalued mappings, Math. Appl. 455, Kluwer, Dordrecht, The Netherlands, 1998. MR1659914 (2000a:54002)

[18] Tolstonogov, A. A., On the structure of the solution set for differential inclusions in Banach spaces, Math. USSR Sbornik 46 (1983), 1-15 (in Russian) (1984), 229-242.

Faculty of Mathematics and Information Science, Warsaw University of Technology, Plac Politechniki 1, 00-661 Warsaw, Poland

E-mail address: fryszko@alpha.mini.pw.edu.pl

URL: http://www.alpha.mini.pw.edu.pl

Faculty of Mathematics and Information Science, Warsaw University of Technology, Plac Politechniki 1, 00-661 Warsaw, Poland

E-mail address: tarz@alpha.mini.pw.edu.pl

$U R L:$ http://www.alpha.mini.pw.edu.pl 